\title{
Beyond antibiotics?
}

\author{
LE Nicolle MD FRCP, Editor-in-Chief
}

$\mathrm{T}_{\mathrm{p}}^{\mathrm{h}}$ he AMMI Canada meeting in March 2006 hosted a symposium exploring the potential alternatives to antibiotics for the prevention and treatment of infection. Four papers summarizing talks from that session are published in this issue of the Journal (1-4). These reviews address the scientific underpinnings for a number of proposed concepts, and summarize the current status of clinical use. The approaches - probiotics, bacteriophage therapy, and manipulation of innate immunity are all intriguing but are still removed from immediate practical applications.

As a prelude to any discussion of antibiotic alternatives, the glorious but troublesome history of antibiotics should be revisited. Why are alternatives needed? As Dr Davies eloquently reminds us, we have, in many respects, lost our way with antimicrobials (1). The spectacular use of antibiotics in some parts of the food industry, progressive evolution of antimicrobial resistance, and current lack of incentives for industry to develop new agents are familiar themes. Inappropriate use, complacency and the drumbeat of corporate profits are the chorus of this tragedy.

Antimicrobial therapy remains effective for most bacterial infections. This efficacy can be preserved, or even salvaged, as bacterial resistance mirrors antibiotic pressure. The prompt development and rapid expansion of antimicrobial resistance and the diminishing development of new antimicrobials are, to a large extent, human failures. The systematic study of our current antimicrobial armamentarium to characterize optimal clinical use, the restriction of nonhuman use of antimicrobials, and rigorous and aggressive stewardship programs for antibiotic use can still contribute to preserving effectiveness. These interventions, however, have been promoted for many years with relatively limited acceptance by health care funders, industry, the research agenda, and perhaps even ourselves in our own practice. So, yes, it is prudent to anticipate a less secure world where antibiotics can no longer sustain the therapeutic triumphs of the past 50 years.

Manipulation of the colonizing human flora, usually with a goal of reconstituting 'normal flora' for the prevention or treatment of infections, has been a holy grail of microbiology for over 100 years. Therapeutic products, now called 'probiotics', are defined as "live microorganisms that, when administered in adequate amount, confer a health benefit on the host". Barriers to the effective development and utilization of probiotics are substantial, starting with the very definition of a 'probiotic' (2). These products are not a single molecule, but rather a complex living collection of microorganisms. How are products standardized? How are they evaluated in clinical trials? The complexity of the human flora will likely mean that new concepts of effectiveness, product development and evaluation need to be framed in evaluating a therapeutic role for probiotics. This complexity is, to some extent, responsible for the primitive knowledge and limited clinical applications of this therapeutic approach, despite prolonged academic and popular interest. It is not yet clear how these challenges will be effectively circumvented.

There are reports of clinical efficacy of some probiotics, particularly in the management of diarrheal diseases, including recurrent Clostridium difficile. However, critical reviews conclude the evidence is still not compelling $(5,6)$, and the experience of clinicians in the field (eg, for relapsing $\mathrm{C}$ difficile) has also not been enthusiastic. Our sketchy knowledge of the normal microbiology of the human body, including what is 'normal', not only raises issues about efficacy, but also of unforeseen consequences. Recent reports of Saccharomyces cerevisiae fungemia in patients treated with a 'Saccharomyces boulardii' preparation to prevent or treat antibiotic-associated diarrhea are cautionary (7).

Bacteriophages are 'viruses' that attack bacteria. Interest in exploiting the therapeutic potential of bacteriophages also has a history of almost 100 years. Clinical initiatives using phage therapy disappeared in North America coincident with the development and introduction of antibiotics, but development and use continued in the former Soviet Union and some eastern European countries (3). There is substantial clinical experience with bacteriophage therapy, particularly for managing diarrhea and wounds. Unfortunately, most published clinical studies are not in English and are thus not readily accessible; furthermore, the clinical trials do not meet current standards for design and evaluation. The active exploration of applications of bacteriophage therapy in the food industry - poultry and dairy cattle, in particular - is resurgent, with inconsistent reports of efficacy (8).

A phage is less complex than a bacterial cell, and product standardization may be more straightforward than probiotics. But bacteriophages are living organisms and can mutate. They elicit an immune response that may limit the durability of activity of a particular phage, and microorganisms can develop resistance to phages. Clarifying the benefits, if any, of phage therapy requires detailed description of the in vivo properties of libraries of phage-bacterium systems. Currently, there is no clear vision of bacteriophages as a meaningful alternative to antibiotics. However, a large and tantalizing body of evidence and experience, including suggestive reports of efficacy in both animals and humans, together with an enthusiastic group of proponents, support continuing critical evaluation of the role of phage therapy. 
Another concept considered at the symposium was innate immunity, which involves the development of products to manipulate human biology, rather than bacteria. While probiotics and bacteriophage therapy have been used clinically for 100 years, exploiting innate immunity is a relatively recent concept. The clinical use of probiotics and phage therapy largely preceded a systematic exploration of the scientific basis for their efficacy. For innate immunity, the scientific knowledge is substantial and rapidly expanding, while clinical applications are only beginning to be developed.

The innate immune system - the initial defense against infections - presents multiple potential targets for the development of agonists, antagonists and adjuvants (4). Animal studies and initial human trials of potential products are underway. But the dark side of manipulation of the immune system is also present; potential therapeutic products may be harmful through overstimulation or blocking of essential molecules or pathways (9). Infecting organisms may also be able to circumvent single target products. Genetic variation seems likely in both efficacy and toxicity for products that act through the manipulation of human biology. Exploration and exploitation of the therapeutic potential of the innate immune system is a story that has just begun.

The concepts described provide a window into a universe of novel anti-infective treatments. New conceptual approaches may be a path to therapeutic triumph, or perhaps subject to chaos theory and unforeseen consequences. Optimism is essential in the exploration of new strategies, together with an enthusiasm for rigorous scientific and clinical knowledge. Critical evaluation must accompany every step of the journey.
Sustained support from individual researchers, funding organizations, industry and regulatory bodies will be necessary for any success. Equally appropriate is continued enthusiasm for programs and research aimed at maintaining the utility of current antibiotics. The development and application of any new products should be considered in the context of the antibiotic experience. Perhaps we are a little wiser, and some of the mistakes of overexuberant and inappropriate use will not be repeated.

\section{REFERENCES}

1. Davies J. Where have all the antibiotics gone? Can J Infect Dis Med Microbiol 2006;17:287-290.

2. Reid G. Probiotics to prevent the need for, and augment the use of, antibiotics. Can J Infect Dis Med Microbiol 2006;17:291-295.

3. Kropinski AM. Phage therapy - Everything old is new again. Can J Infect Dis Med Microbiol 2006;17:297-306.

4. Rosenthal KL. Tweaking innate immunity: The promise of innate immunologicals as anti-infectives. Can J Infect Dis Med Microbiol 2006;17:307-314.

5. Sazawal S, Hiremath G, Dhingra U, Malik P, Deb S, Black RE. Efficacy of probiotics in prevention of acute diarrhoea: A metaanalysis of masked, randomised, placebo-controlled trials. Lancet Infect Dis 2006;6:374-82.

6. Johnston BC, Supina AL, Vohra S. Probiotics for pediatric antibiotic-associated diarrhea: A meta-analysis of randomized placebo-controlled trials. CMAJ 2006;175:377-83.

7. Munoz P, Bouza E, Cuenca-Estrella M, et al. Saccharomyces cerevisiae fungemia: An emerging infectious disease. Clin Infect Dis 2005; $40: 1625-34$.

8. Skurnik M, Strauch E. Phage therapy: Facts and fiction. Int J Med Microbiol 2006;296:5-14.

9. Suntharalingam G, Perry MR, Ward S, et al. Cytokine storm in a phase 1 trial of the anti-CD28 monoclonal antibody TGN1412. N Engl J Med 2006;355:1018-28. 


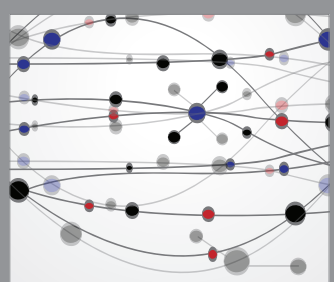

The Scientific World Journal
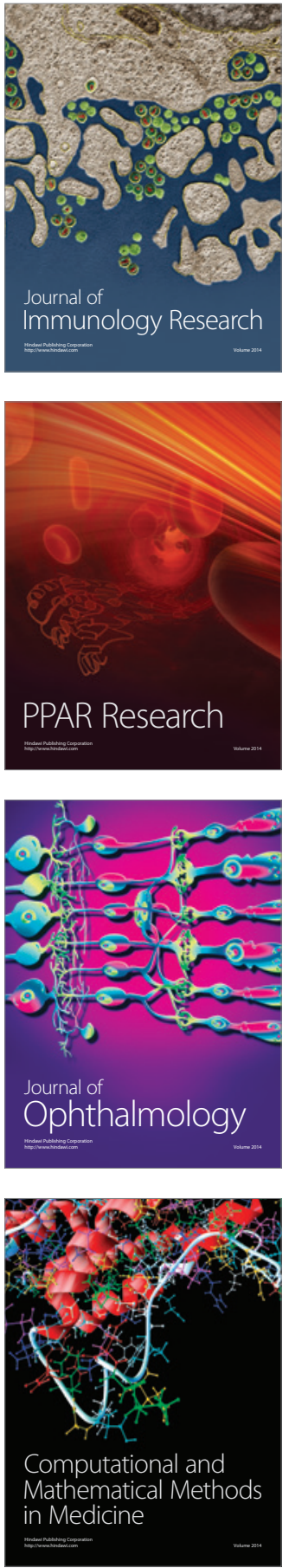

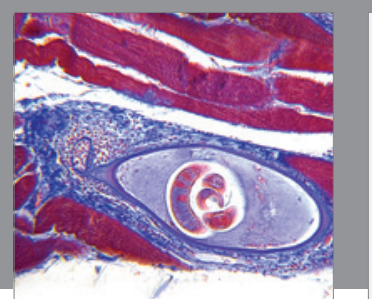

Gastroenterology Research and Practice

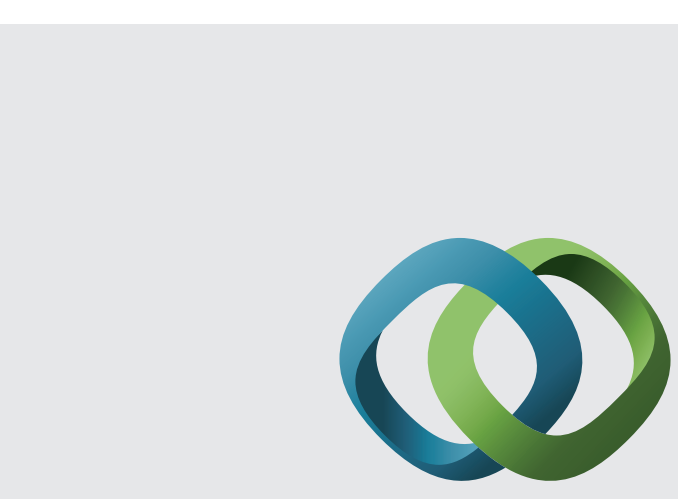

\section{Hindawi}

Submit your manuscripts at

http://www.hindawi.com
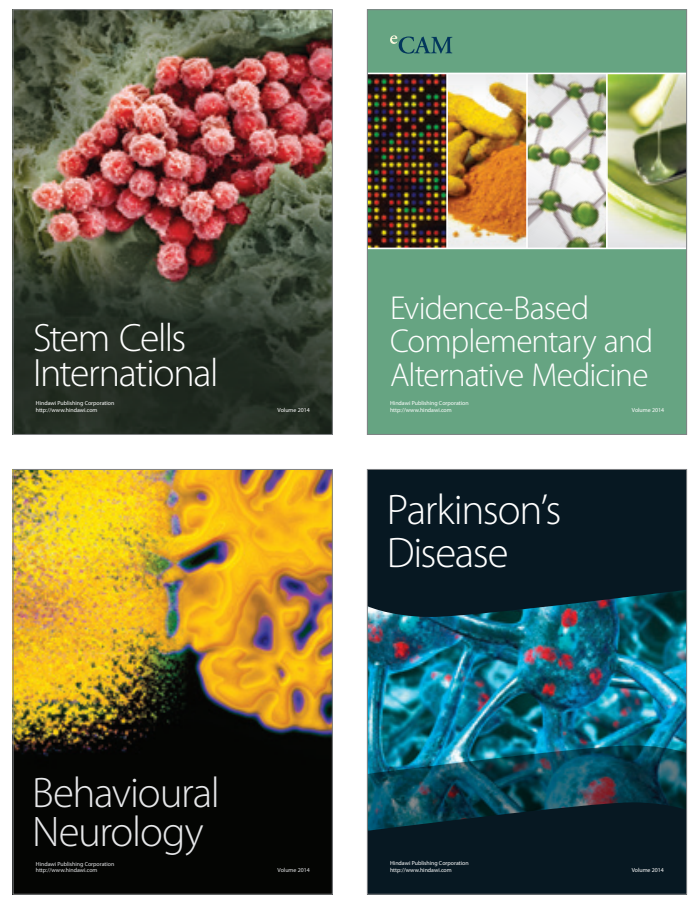
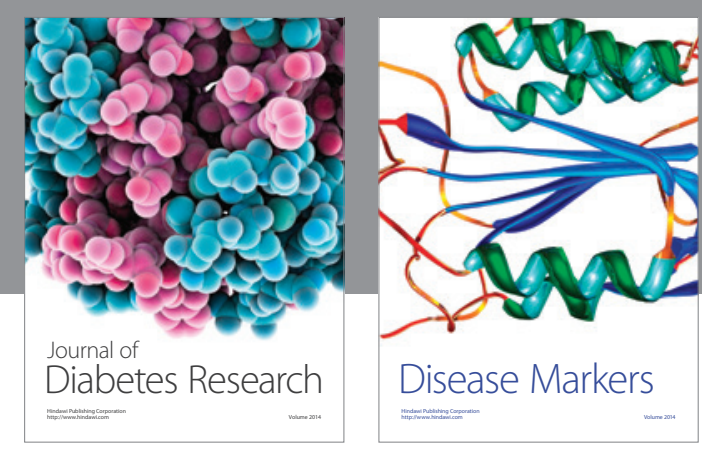

Disease Markers
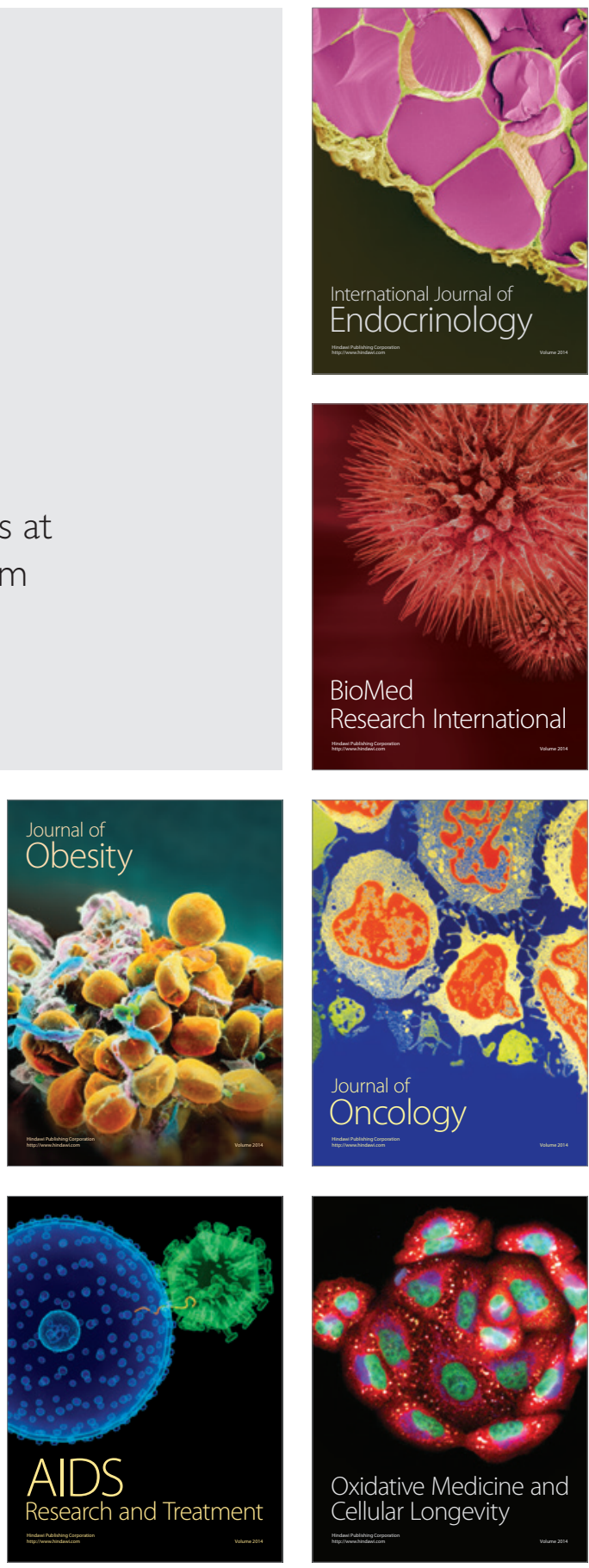